\title{
An updated tree of Y-chromosome Haplogroup 0 and revised phylogenetic positions of mutations P164 and PK4
}

\author{
Shi Yan, Chuan-Chao Wang, Hui Li, Shi-Lin Li, Li Jin and The Genographic Consortium
}

European Journal of Human Genetics (2011) 19, 1018; doi:10.1038/ejhg.2011.116

Correction to: European Journal of Human Genetics (2011) 19, 1013-1015; doi:10.1038/ejhg.2011.64; published online 20 April 2011

The list of Genographic Consortium members listed in the Acknowledgements section was incorrect. The correct list is published below. The authors would like to apologise for this error.

The Genographic Consortium includes Janet S Ziegle (Applied Biosystems, Foster City, CA, USA); Pandikumar Swamikrishnan (IBM, Somers, NY, USA); Asif Javed, Laxmi Parida, Daniel E Platt and Ajay K Royyuru (IBM, Yorktown Heights, NY, USA); Lluis Quintana-Murci (Institut Pasteur, Paris, France); R John Mitchell (La Trobe University, Melbourne, Victoria, Australia); Syama Adhikarla, ArunKumar GaneshPrasad, Ramasamy Pitchappan, Kavitha Valampuri John and Arun Varatharajan Santhakumari (Madurai Kamaraj University, Madurai, Tamil Nadu, India); Christoff J Erasmus, Angela Hobbs and Himla Soodyall (National Health Laboratory Service, Johannesburg, South Africa); Doron M Behar
(Rambam Medical Center, Haifa, Israel); Elena Balanovska and Oleg Balanovsky (Research Centre for Medical Genetics, Russian Academy of Medical Sciences, Moscow, Russia); Chris Tyler-Smith (The Wellcome Trust Sanger Institute, Hinxton, UK); Daniela R Lacerda and Fabrício R Santos (Universidade Federal de Minas Gerais, Belo Horizonte, Minas Gerais, Brazil); Pedro Paulo Vieira (Universidade Federal do Rio de Janeiro, Rio de Janeiro, Brazil); Jaume Bertranpetit, David Comas, Begoña Martínez-Cruz and Marta Melé (Universitat Pompeu Fabra, Barcelona, Spain); Christina J Adler, Alan Cooper, Clio SI Der Sarkissian and Wolfgang Haak (University of Adelaide, South Australia, Australia); Matthew E Kaplan and Nirav C Merchant (University of Arizona, Tucson, AZ, USA); Colin Renfrew (University of Cambridge, Cambridge, UK); Andrew C Clarke and Elizabeth A Matisoo-Smith (University of Otago, Dunedin, New Zealand); Matthew C Dulik, Jill B Gaieski, Amanda C Owings and Theodore G Schurr (University of Pennsylvania, Philadelphia, PA, USA); David F Soria Hernanz and R Spencer Wells (National Geographic Society, Washington, DC, USA).

\section{Extended extraocular phenotype of PROM1 mutation in kindreds with known autosomal dominant macular dystrophy}

Francesca I Arrigoni, Mar Matarin, Pamela J Thompson, Michel Michaelides, Michelle E McClements, Elizabeth Redmond, Lindsey Clarke, Elizabeth Ellins, Saifullah Mohamed, Ian Pavord, Nigel Klein, David M Hunt, Anthony T Moore, Julian Halcox and Sanjay M Sisodiya

European Journal of Human Genetics (2011) 19, 1018; doi:10.1038/ejhg.2011.73

Correction to: European Journal of Human Genetics (2011) 19, The authors would like to apologize for this error. 131-137; doi:10.1038/ejhg.2010.147; published online 22 September 2010

In this article published online on 22 September 2010, the name of one of the authors, Nigel Klein, was omitted. 